\title{
Divergência genética entre acessos de açaizeiro fundamentada em descritores morfoagronômicos
}

\author{
Maria do Socorro Padilha de Oliveira ${ }^{(1)}$, Daniel Furtado Ferreira( ${ }^{(2)}$ e João Bosco dos Santos ${ }^{(3)}$
}

\begin{abstract}
(1)Embrapa Amazônia Oriental, Caixa Postal 48, CEP 66095-100 Belém, PA. E-mail: spadilha@cpatu.embrapa.br (2)Universidade Federal de Lavras (UFLA), Dep. de Ciências Exatas, Caixa Postal 3037, CEP 37200-000 Lavras, MG E-mail: dff@ufla.br (3)UFLA, Dep. de Biologia. E-mail: jbsantos@ufla.br
\end{abstract}

\begin{abstract}
Resumo - Este trabalho teve como objetivo estimar a divergência genética entre acessos de açaizeiro conservados na coleção de germoplasma da Embrapa Amazônia Oriental, em Belém, PA, por meio de descritores morfoagronômicos. A avaliação foi realizada em 87 acessos, com base em 22 caracteres: sete relativos à planta, três à floração, três a frutos e nove à produção de frutos, no período de 1995 a 2001. Foram efetuadas análises univariadas e multivariadas, com estimativas das dissimilaridades obtidas pela distância euclidiana média padronizada, e formação dos agrupamentos obtida pelos métodos UPGMA e Tocher. Os acessos apresentaram alto índice de variação na maioria dos caracteres. As distâncias genéticas entre os pares de acessos variaram de 0,09 a 1,87, com média de 1,39. O método UPGMA dividiu os acessos em cinco grupos, enquanto o de Tocher formou 24 agrupamentos. Os cinco acessos indicados como mais divergentes devem compor programas de intercruzamentos, para obtenção de genótipos superiores.
\end{abstract}

Termos para indexação: Euterpe oleracea, variabilidade, análise multivariada, agrupamento, distância genética.

\section{Genetic divergence among accessions of assai palm based on morphoagronomic descriptors}

\begin{abstract}
The objective of this research was to estimate the genetic divergence among accessions of the assai palm, sampled in the germplasm collection of Embrapa Amazônia Oriental based on morphoagronomic traits, at Belém, PA. The 22 morphoagronomic traits were evaluated in 87 accessions, from 1995 to 2001. Among the 22 traits, seven were relative to the plant, three to the flowering, three to the fruit, and nine to the fruit production. The traits were analyzed using univariate and multivariate analysis. The estimates of the dissimilarities were obtained by the average Euclidian distance with standardized data, and the groupings by UPGMA and Tocher methods. The accessions presented high variation index in the majority of the characters. Genetic distances among accession pair varied from 0.09 to 1.87 , with average of 1.39 . The method UPGMA divided the accessions into five groups, while the Tocher method formed 24 groupings. The five accessions indicated as more divergent must be used in breeding programs aiming at the obtention of superior genotypes.
\end{abstract}

Index terms: Euterpe oleracea, variability, multivariate analysis, grouping, genetic distance.

\section{Introdução}

$\mathrm{O}$ açaizeiro (Euterpe oleracea Mart.) é uma importante espécie perene da Amazônia que, nos últimos anos, vem despertando interesse no cultivo para produção de frutos para o mercado de polpa congelada (Oliveira \& Farias Neto, 2006). Atualmente, a expansão dos mercados nacional e internacional da polpa processada dessa palmeira está em torno de 30 e $16 \%$ ao ano, respectivamente, o que evidencia a necessidade da ampliação de áreas plantadas com sementes de qualidade reconhecida. Contudo, há escassez de informações sobre seu germoplasma que possam orientar programas de melhoramento genético, na obtenção de cultivares desejáveis para a produção de frutos, especialmente quanto à divergência genética.

Estudos que envolvem a divergência em plantas perenes têm sido realizados, freqüentemente, com base em descritores botânicos, morfológicos e agronômicos, por não apresentarem custos elevados (Dias et al., 1997). No entanto, as interpretações desses dados têm, habitualmente, sido feitas por análises univariadas, o que gera dificuldades na obtenção das estimativas de divergência e, conseqüentemente, na seleção de 
indivíduos desejáveis para intercruzamentos. Assim, as técnicas multivariadas têm se mostrado úteis, por avaliar o indivíduo em vários aspectos e proporcionar uma visão holística de cada acesso (Cruz et al., 2004).

Análises de agrupamentos têm sido empregadas, com freqüência, na visualização e interpretação da divergência genética, com base em caracteres morfológicos e agronômicos em plantas perenes (Dias et al., 1997; Ribeiro et al., 1999; Araujo et al., 2002; Alves et al., 2003; Martel et al., 2003; Sousa, 2003). Para as variáveis quantitativas, a medida de dissimilaridade sugerida é a distância euclidiana média padronizada, quando o germoplasma encontra-se instalado sem casualização e ou sem controle local (Cruz et al., 2004). A diversidade vem sendo quantificada em germoplasma de algumas espécies perenes, nativas da Amazônia, como o cacau (Dias et al., 1997), o cupuaçu (Araujo et al., 2002; Alves et al., 2003) e o guaraná (Nascimento Filho et al., 2001), e tem fornecido importantes informações para os programas de melhoramento genético. Para o germoplasma de palmeiras, esses estudos têm sido raros (Ribeiro et al., 1999; Martel et al., 2003) e há apenas um registro para a espécie em questão (Gazel Filho \& Lima, 2006).

Este trabalho teve como objetivo quantificar a divergência genética entre acessos de açaizeiro, com base em descritores morfoagronômicos para orientar programas de melhoramento dessa espécie.

\section{Material e Métodos}

Utilizaram-se dados de 87 acessos, da coleção de germoplasma de açaizeiro da Embrapa Amazônia Oriental, localizada em Belém, PA (1²7'21"S, 48³0'16"W e 10,8 m de altitude), que apresentaram frutificação em pelo menos uma planta, no período de 1995 a 2001 (Tabela 1). O clima local corresponde ao tipo quente e úmido $\left(\mathrm{Af}_{\mathrm{i}}\right)$, caracterizado por Köppen, por possuir uma estação chuvosa com precipitação média de $2.740 \mathrm{~mm}$, com temperatura média de $26^{\circ} \mathrm{C}$ e umidade relativa do ar próxima de $90 \%$.

Essa coleção foi estabelecida por meio de coletas direcionadas, em 21 locais (procedências) que abrangem o Pará, Amapá e Maranhão, realizadas pela retirada de um cacho de matrizes, com características desejáveis para a produção de frutos, registradas como Euterpe oleracea (Lima \& Costa, 1991). Na coleção, os acessos encontram-se representados por número variável de plantas, dispostas em linhas ao acaso, no espaçamento de $5 \times 3 \mathrm{~m}$, que correspondem a famílias de meios-irmãos.
Os caracteres quantitativos mensurados foram: 17 morfológicos (sete relativos à planta, quatro à floração, seis a frutos) e 11 agronômicos, que fazem parte da lista preliminar de descritores (Oliveira, 1998). Os caracteres foram obtidos de todas as plantas representantes de cada acesso, e o número de caracteres variou de 1 a 8 por acesso, em 247 plantas.

Os caracteres da planta foram avaliados em abril de 1997 e envolveram: número de estipe por planta; número de estipe em frutificação por planta; altura do estipe mais velho da planta; número de folhas do estipe mais velho da planta; comprimento da bainha foliar, da folha mais externa do estipe mais velho da planta; circunferência do estipe mais velho da planta; e comprimento de cinco entrenós do estipe mais velho da planta, conforme Oliveira (1998).

Os caracteres de floração foram observados em uma inflorescência, por planta, de cada acesso, a partir da abertura da bráctea, e são: duração da fase masculina; intervalo entre fases; duração da fase feminina; duração total da floração.

Os caracteres de frutos foram obtidos de três cachos, por planta, tendo sido retirados ao acaso cinco frutos por cacho, tendo-se observado: diâmetro longitudinal e transversal do fruto; peso do fruto, da semente e da parte comestível; e rendimento da parte comestível por fruto.

Tabela 1. Identificação dos 87 acessos de açaizeiro, da coleção de germoplasma da Embrapa Amazônia Oriental, utilizados no estudo ${ }^{(1)}$.

\begin{tabular}{|c|c|c|}
\hline Procedência & $\begin{array}{l}\text { Local de } \\
\text { coleta }^{(1)}\end{array}$ & $\begin{array}{c}\text { № de } \\
\text { acessos }\end{array}$ \\
\hline Benfica, PA & Pomar caseiro & 2 \\
\hline Benevides, PA & Pomar caseiro e propriedade rural & 4 \\
\hline Mosqueiro, PA & Pomar caseiro & 1 \\
\hline $\mathrm{St}^{\circ}$ Antônio do Tauá, PA & Propriedade rural & 5 \\
\hline Chaves, PA & População natural & 12 \\
\hline Muaná, PA & Propriedade rural & 11 \\
\hline Breves, PA & População natural e propriedade rural & 25 \\
\hline Gurupá, PA & Pomar caseiro & 3 \\
\hline Melgaço, PA & Propriedade rural & 1 \\
\hline Santarém, PA & Propriedade rural e pomar caseiro & 2 \\
\hline Prainha, PA & Pomar caseiro & 1 \\
\hline Cametá, PA & População natural & 1 \\
\hline Oiapoque, AP & População natural e pomar caseiro & 6 \\
\hline Macapá, AP & Pomar caseiro & 2 \\
\hline Maracaçumé, MA & Propriedade rural & 2 \\
\hline $\mathrm{St}^{\mathrm{a}}$ Luzia, MA & Propriedade rural & 1 \\
\hline Turiaçu, MA & População natural & 2 \\
\hline $\mathrm{St}^{\mathrm{a}}$ Inêz, MA & População natural & 1 \\
\hline Mirinzal, MA & População natural & 1 \\
\hline Guimarães, MA & População natural & 2 \\
\hline Alcântara, MA & População natural e pomar caseiro & 2 \\
\hline Total & & 87 \\
\hline
\end{tabular}

${ }^{(1)}$ Fonte (Lima \& Costa, 1991). 
Os caracteres agronômicos foram avaliados no período de sete anos (1995 a 2001) e contabilizados no final de cada ano, exceto o caráter dias após a polinização, obtido de uma inflorescência por planta. Foram observados: dias após a polinização; número de anos em produção; número de meses em produção; peso total do cacho; peso de frutos por cacho; rendimento de frutos por cacho; número de ráquilas por cacho; comprimento da ráquis do cacho; peso de cem frutos; número total de cachos; produção total de frutos.

Os caracteres foram submetidos às análises de variância univariada (ANOVA) - apesar de a coleção não ter sido instalada em delineamento experimental, apresenta aleatorização das linhas e das plantas representantes dos acessos - e multivariada. A ANOVA foi realizada apenas para constatar a existência de variação entre os acessos, tendo-se considerado o modelo inteiramente casualizado, com 87 tratamentos (acessos), e de uma a oito repetições (plantas), pelo procedimento GLM do SAS v.8.1 (SAS Institute, 2000). A análise multivariada empregada foi a de agrupamento, efetuada para os 22 caracteres de maior importância para a produção de frutos (Oliveira et al., 2006), por dois mé- todos: o hierárquico aglomerativo de ligação média não ponderada (UPGMA) e o não hierárquico de otimização de Tocher, ambos com base na distância euclidiana média padronizada $\left(\mathrm{d}_{\mathrm{ii}}\right)$, obtida no programa GQMOL (Cruz \& Schuster, 2004). Essa distância foi escolhida pelo fato de os dados terem sido coletados em experimento que não envolve delineamento experimental. $\mathrm{O}$ método UPGMA foi gerado pelo programa NTSYS-pc 2,1 (Rohlf, 2000), e o método de otimização de Tocher pelo GQMOL (Cruz \& Schuster, 2004).

\section{Resultados e Discussão}

A maioria dos caracteres revelou diferenças altamente significativas entre acessos, pelo teste $\mathrm{F}(\mathrm{p} \leq 0,01)$, exceto para a duração das fases feminina $(\mathrm{p} \leq 0,05)$ e masculina, e para o rendimento de polpa por fruto (Tabela 2). Em média, os acessos apresentaram características desejáveis quanto à produção de frutos com bom perfilhamento (cinco estipes por planta) e alto rendimento de frutos por cacho $(71 \%)$ e da parte comestível por fruto $(23,3 \%)$, mas exibiram baixos valores quanto ao número de cachos $(3,5)$ e produção de frutos por planta

Tabela 2. Resumo das análises de variância, obtidas para os 28 caracteres morfoagronômicos avaliados nos 87 acessos de açaizeiro.

\begin{tabular}{|c|c|c|c|c|}
\hline \multirow[t]{2}{*}{ Caracteres } & \multicolumn{2}{|c|}{ Quadrados médios } & \multirow[t]{2}{*}{ Média } & \multirow{2}{*}{$\begin{array}{l}\text { CV } \\
(\%)\end{array}$} \\
\hline & Acessos & Erro & & \\
\hline Número de estipe por planta (unidade) & $14,170^{* *}$ & 5,211 & 5,05 & 45,18 \\
\hline Número de estipe em frutificação por planta (unidade) & $7,377^{* *}$ & 2,482 & 3,85 & 40,91 \\
\hline Altura do estipe (m) & $4,006^{* *}$ & 0,271 & 6,34 & 8,21 \\
\hline Número de folhas (unidade) & $1,210^{* *}$ & 0,715 & 10,75 & 7,87 \\
\hline Comprimento da bainha foliar (m) & $0,016^{* *}$ & 0,005 & 1,04 & 6,85 \\
\hline Circunferência do estipe $(\mathrm{cm})$ & $42,175^{* *}$ & 13,426 & 32,11 & 11,41 \\
\hline Comprimento de cinco entrenós $(\mathrm{cm})$ & $320,440^{* *}$ & 91,131 & 59,67 & 16,00 \\
\hline Duração da fase masculina (dias) & $3,852^{\text {ns }}$ & 2,929 & 11,20 & 15,54 \\
\hline Intervalo entre fases (dias) & $3,755^{* *}$ & 1,863 & 2,02 & 67,55 \\
\hline Duração da fase feminina (dias) & $0,763^{*}$ & 0,557 & 4,18 & 17,85 \\
\hline Duração total da floração (dias) & $5,716^{* *}$ & 2,994 & 17,19 & 10,06 \\
\hline Diâmetro longitudinal do fruto (mm) & $1,173^{* *}$ & 0,333 & 11,89 & 4,86 \\
\hline Diâmetro transversal do fruto (mm) & $1,600^{* *}$ & 0,388 & 13,63 & 4,57 \\
\hline Peso do fruto $(\mathrm{g})$ & $0,152^{* *}$ & 0,034 & 1,41 & 13,09 \\
\hline Peso da parte comestível (g) & $0,010^{* *}$ & 0,004 & 0,32 & 19,52 \\
\hline Peso da semente $(\mathrm{g})$ & $0,091^{* *}$ & 0,024 & 1,08 & 14,34 \\
\hline Rendimento da parte comestível por fruto $(\%)$ & $10,707^{\mathrm{ns}}$ & 8,350 & 23,27 & 12,42 \\
\hline Dias após a polinização (dias) & $883,153^{* *}$ & 340,786 & 181,01 & 10,20 \\
\hline Número de anos em produção (unidade) & $3,642^{* *}$ & 1,500 & 4,40 & 27,85 \\
\hline Número de meses em produção (unidade) & $0,565^{* *}$ & 0,369 & 1,89 & 32,20 \\
\hline Número total de cachos (unidade) & $3,360^{* *}$ & 2,107 & 3,49 & 41,64 \\
\hline Produção total de frutos $(\mathrm{kg})$ & $20,923^{* *}$ & 12,300 & 6,72 & 52,19 \\
\hline Peso total do cacho $(\mathrm{kg})$ & $0,823^{* *}$ & 0,344 & 2,53 & 23,19 \\
\hline Peso de frutos por cacho $(\mathrm{kg})$ & $0,559^{* *}$ & 0,245 & 1,88 & 26,38 \\
\hline Rendimento de frutos por cacho (\%) & $43,945^{* *}$ & 20,558 & 71,04 & 6,38 \\
\hline Número de ráquilas por cacho (unidade) & $228,053^{* *}$ & 111,011 & 98,93 & 10,65 \\
\hline Comprimento da ráquis do cacho $(\mathrm{cm})$ & $114,616^{* *}$ & 60,614 & 52,25 & 14,90 \\
\hline Peso de 100 frutos $(\mathrm{g})$ & $985,841^{* *}$ & 172,161 & 145,39 & 9,02 \\
\hline
\end{tabular}

${ }^{n s}$ Não-significativo. ${ }^{*} \mathrm{e}^{* *}$ Significativo pelo teste $\mathrm{F}$, a 5 e $1 \%$ de probabilidade, respectivamente. 
por ano $(7,6 \mathrm{~kg})$, o que indica que esses últimos caracteres sofreram pouca influência de seleção. Oliveira et al. (1998) também constataram alta variação para a produção de frutos por planta, ao analisar 20 acessos dessa coleção $(0,1$ a $50,9 \mathrm{~kg}$ de frutos por planta por ano). Portanto, indivíduos dentro de acessos que apresentem valores acima da média, devem ser promissores para a produção de frutos.

Os coeficientes de variação foram bastante variáveis, com os menores valores registrados para o diâmetro do fruto, rendimento de frutos por cacho, comprimento da bainha foliar, número de folhas, altura do estipe, peso de cem frutos, duração total da floração, dias após a polinização, número de ráquilas por cacho e circunferência do estipe $(\mathrm{CV} \leq 10,41 \%)$.

Os caracteres número de meses em produção, número de estipe em frutificação, de cachos e de estipes por planta, produção total de frutos e intervalo entre fases de floração exibiram valores acima de $30 \%$. Tais resultados eram esperados, por serem os acessos famílias de meios-irmãos e, portanto, com representatividade variável de variabilidade. Ressalte-se, ainda que, apesar de os caracteres terem sido mensurados em experimento sem controle local, detectaram-se diferenças significativas entre os acessos para a maioria dos caracteres, com evidência de ampla variabilidade entre esses caracteres, o que está de acordo com Oliveira et al. (1998), que estudaram outros acessos dessa coleção. Vargas \& Blanco (2000) e Sugimura et al. (1997), quando avaliaram caracteres morfológicos e de frutificação em cultivares de coqueiro, também obtiveram resultados semelhantes. O mesmo fato foi constatado por Alves et al. (2003), ao analisar a variação entre clones de cupuaçuzeiro, em condições experimentais similares às deste estudo, com base em caracteres morfológicos e agronômicos.

As distâncias genéticas entre os pares de acessos variaram de 0,09 a 1,87, com média de 1,39. A menor distância foi obtida entre os acessos 26 (Chaves, PA) e 49 (Breves, PA), enquanto os acessos 68 (Breves, PA) e 78 (Oiapoque, AP) formaram o par mais divergente. Mais da metade dos pares de acessos $(52,87 \%)$ apresentou distâncias genéticas médias iguais ou acima da média geral, o que evidencia que grande parte deles detém acentuada divergência. Como as distâncias genéticas foram obtidas com base nos 22 caracteres de maior importância para a produção de frutos (Oliveira et al., 2006), acredita-se que essas informações possam ser úteis para programas de melhoramento genético do açaizeiro que utilizam hibridação, por fornecer subsídios para restringir o número de genitores, na formação da população segregante. Entretanto, devem ser acompanhadas pela avaliação da capacidade geral e específica de combinação, para poucos acessos divergentes, uma vez que os acessos são formados por sementes de polinização livre havendo apenas controle maternal.

A distribuição das distâncias genéticas, feita com base nos 3.741 pares gerados entre os acessos, encontra-se representada na Figura 1. Observa-se que as seis primeiras classes foram formadas por poucos pares, detentores de distâncias entre 0,09 e 0,90, ou seja, acessos geneticamente próximos, e que a maioria dos pares $(92 \%)$ ficou concentrada nas classes cujas distâncias variaram de 1,06 a 1,71, o que caracteriza forte assimetria à direita e, conseqüentemente, aloca os pares de acessos com alta divergência genética. Esse tipo de distribuição revela que os pares de acessos analisados mostraramse bastante divergentes, em relação aos 22 descritores selecionados. Tal resultado demonstra que a seleção realizada para a produção de frutos, durante a coleta do germoplasma estudado, foi efetiva, e que se pode esperar ganhos genéticos consideráveis com a seleção fenotípica de indivíduos, nessa coleção.

O dendrograma gerado pelo método UPGMA apresentou valor cofenético alto e significativo $(r=0,88$, $\mathrm{p} \leq 0,0001$ ), o que mostra fidelidade na representação do conjunto de dados. Nenhum acesso formou grupo divergente isolado, mas sim vários grupos com subgrupos que continham três a quatro acessos, com distâncias semelhantes entre si, mas como o ponto de corte foi

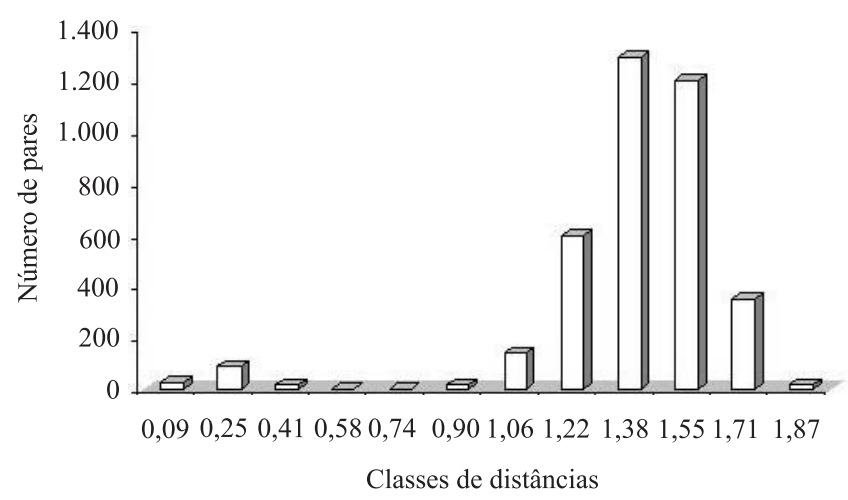

Figura 1. Distribuição de freqüência das estimativas de distância genética, obtidas entre os pares dos 87 acessos de açaizeiro, com base nos 22 descritores selecionados. 
feito com base na média geral $\left(\mathrm{dg}_{\mathrm{E}}=1,39\right)$ foi possível a separação dos acessos em cinco grupos (Figura 2).

Os dois primeiros grupos (a) e (b) foram formados por 11 acessos: $97,59,35,9,80,46,23,96,57,34$ e 8 ; e $78,45,22,110,76,44,21,95,56,33$ e 7 , respectivamente, considerados como os mais divergentes. Enquanto o último grupo (e) foi constituído por 33 acessos, possivelmente os menos divergentes. De modo geral, houve pouca tendência de os acessos da mesma procedência ficarem inseridos no mesmo grupo, o que evidencia maior variação dentro das procedências que entre elas. Gazel Filho \& Lima (2006), ao analisar caracteres morfoagronômicos, também obtiveram a formação de poucos grupos com 39 matrizes de açaizeiro, coletadas em vários locais. A formação de poucos grupos tem sido relatada em outras palmeiras (Vargas \& Blanco, 2000; Martel et al., 2003).

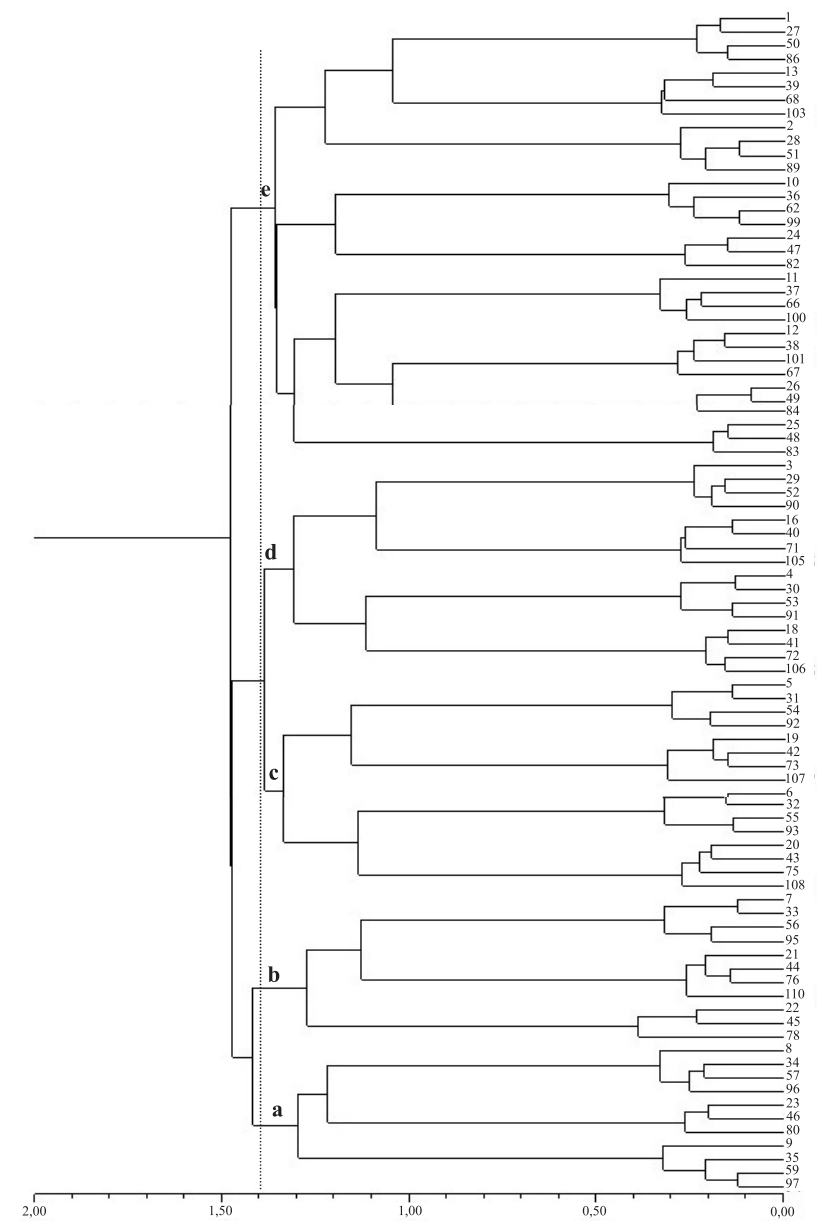

Figura 2. Dendrograma gerado pelo método UPGMA, a partir das distâncias genéticas obtidas nos 22 descritores selecionados, com a relação entre os 87 acessos de açaizeiro. Ponto de corte $\left(\operatorname{dg}_{\mathrm{E}}=1,39\right)$.
O método de Tocher revelou a formação de 24 grupos distintos (Tabela 3) os quais, com exceção do grupo 24 , formado por um acesso (78), foram constituídos por poucos acessos (dois a quatro). As distâncias intragrupos variaram de 0,18 a 0,30 , por conseguinte os grupos 18, 21 e 22 destacaram-se como possuidores das maiores divergências e devem conter acessos distintos. Pelas distâncias intergrupos foram constadas maiores dissimilaridades entre os grupos 3 e $24(1,78)$ e o 18 e 24 (1,76), com acessos bem divergentes $(28,51,89,2$, 13, 39, 68, 103 e 78). Assim como no método UPGMA, este método também não organizou os acessos por local de coleta, o que reforça novamente a evidência de grande divergência entre acessos da mesma procedência. Esses resultados demonstram que cruzamentos entre os acessos mais divergentes podem proporcionar aumento na variabilidade e, possivelmente, a obtenção de indivíduos superiores.

Ao comparar os agrupamentos obtidos pelos dois métodos, percebe-se que, com exceção dos últimos grupos, formados no método de Tocher, houve alguma semelhança nos demais. Porém, se o ponto de corte no dendrograma fosse efetuado à distância igual a 1,00, o número de agrupamentos seria quase o mesmo, assim como as suas constituições, com mudança apenas na ordem seqüencial dos grupos. Outros trabalhos com

Tabela 3. Grupos formados pelo método de otimização de Tocher, com base nas distâncias euclidianas, obtidas para os 22 caracteres selecionados nos 87 acessos de açaizeiro ${ }^{(1)}$.

\begin{tabular}{|c|c|}
\hline Grupos & Acessos \\
\hline 1 & 26 (Chaves), 49 (Breves), 84 (Gurupá) \\
\hline 2 & 7 (Benevides), 33 (Muaná), 56 (Breves), 95 (Maracaçumé) \\
\hline 3 & 28 (Muaná), 51 (Breves), 89 (Breves), 2 (Benfica) \\
\hline 4 & 59 (Breves), 97 ( $\mathrm{St}^{\mathrm{a}}$ Luzia), 35 (Muaná), 9 ( $\mathrm{St}^{\circ}$ Antônio Tauá) \\
\hline 5 & 62 (Breves), 99 (Turiaçu), 36 (Muaná), 10 ( St $^{\circ}$ Antônio Tauá) \\
\hline 6 & 4 (Benevides), 30 (Muaná), 53 (Breves), 91 (Santarém) \\
\hline 7 & 55 (Breves), 93 (Santarém), 32 (Muaná), 6 (Mosqueiro) \\
\hline 8 & 5 (Benevides), 31 (Muaná), 54 (Breves), 92 (Prainha) \\
\hline 9 & 16 (Chaves), 40 (Breves), 71 (Macapá), 105 (Guimarães) \\
\hline 10 & 44 (Breves), 76 (Oiapoque), 21 (Chaves), 110 (Cametá) \\
\hline 11 & 18 (Chaves), 41 (Breves), 72 (Oiapoque), 106 (Guimarães) \\
\hline 12 & 24 (Chaves), 47 (Breves), 82 (Oiapoque) \\
\hline 13 & 25 (Chaves), 48 (Breves), 83 (Gurupá) \\
\hline 14 & 42 (Breves), 73 (Oiapoque), 19 (Chaves), 107 (Alcântara) \\
\hline 15 & 50 (Breves), 86 (Gurupá), 27 (Chaves), 1 (Benfica) \\
\hline 16 & 12 ( $\mathrm{St}^{\circ}$ Antônio Tauá), 38 (Muaná), 101 ( $\mathrm{St}^{\mathrm{a}}$ Inêz), 67 (Breves) \\
\hline 17 & 29 (Muaná), 52 (Breves), 90 (Melgaço), 3 (Benevides) \\
\hline 18 & 13 (Chaves), 39 (Breves), 68 (Breves), 103 (Mirinzal) \\
\hline 19 & 20 (Chaves), 43 (Breves), 75 (Macapá), 108 (Alcântara) \\
\hline 20 & 23 (Chaves), 46 (Breves), 80 (Oiapoque) \\
\hline 21 & 34 (Muaná), 57 (Breves), 96 (Maracaçumé), 8 ( $\mathrm{St}^{\circ}$ Antônio Tauá) \\
\hline 22 & 37 (Muaná), 66 (Breves), 100 (Turiaçu), 11 (St Antônio Tauá) \\
\hline 23 & 22 (Chaves), 45 (Breves) \\
\hline 24 & 78 (Oiapoque) \\
\hline
\end{tabular}

${ }^{(1)}$ Localidades entre parênteses representam a procedência do acesso; maior distância entre os mínimos: 0,37. 
espécies arbóreas relatam a coincidência na formação de alguns agrupamentos (Dias et al., 1997; Nascimento Filho et al., 2001; Alves et al., 2003; Sousa, 2003). Com base nessas afirmações, pode-se considerar cinco acessos como os mais divergentes: $78,45,22,96$ e 8 .

\section{Conclusões}

1. Os acessos de açaizeiro, conservados na coleção de germoplasma da Embrapa Amazônia Oriental, possuem elevada variação para a maioria dos caracteres morfoagronômicos analisados.

2. A quantificação da divergência, com base nos 22 descritores selecionados, agrupa com consistência os acessos e permite a formação de, pelo menos, cinco grupos no método UPGMAe de 24 no método de Tocher.

3. Os cinco acessos de açaizeiro mais divergentes, com base nos métodos UPGMA e Tocher, devem ser indicados para programas de hibridação para a obtenção de populações segregantes e, possivelmente, de genótipos superiores.

\section{Referências}

ALVES, R.M.; GARCIA, A.A.F.; CRUZ, E.D.; FIGUEIRA, A. Seleção de descritores botânico-agronômicos para caracterização de germoplasma de cupuaçuzeiro. Pesquisa Agropecuária Brasileira, v.38, p.807-818, 2003.

ARAUJO, D.G. de; CARVALHO, S.P.; ALVES, R.M. Divergência genética entre clones de cupuaçuzeiro (Theobroma grandiflorum Willd. ex Spreng. Schum.). Ciência e Agrotecnologia, v.26, p.1321, 2002.

CRUZ, C.D.; REGAZZI, J.A.; CARNEIRO, P.C.S. Divergência genética. In: CRUZ, C.D.; REGAZZI, J.A.; CARNEIRO, P.C.S. (Ed.). Modelos biométricos aplicados ao melhoramento genético. Viçosa: UFV, 2004. v.1, p.377-413.

CRUZ, C.D.; SCHUSTER, I. GQMOL: aplicativo computacional para análise de dados moleculares e de suas associações com caracteres quantitativos. Versão 2.1. Viçosa, MG: UFV, 2004. Disponível em: <http://www.ufv.br/dbg/gqmol/gqmol.htm>. Acesso em: 30 mar. 2007.

DIAS, L.A. dos S.; KAGEYAMA, P.Y.; CASTRO, G.C.T. Divergência genética multivariada na preservação de germoplasma de cacau (Theobroma cacao L.). Agrotrópica, v.9, p.29-40, 1997.

GAZEL FILHO, A.B.; LIMA, J.A. de S. Diversidade genética de matrizes de açaizeiro (Euterpe oleracea Mart.). In: FRAZÃO, D.A.C.; HOMMA, A.K.O.; VIÉGAS, I. de J.M. (Ed.). Contribuição ao desenvolvimento da fruticultura da Amazônia. Belém: Embrapa Amazônia Oriental, 2006. p.69-72.
LIMA, R.R.; COSTA, J.P.C. da. Registro de introduções de plantas de cultura pré-colombiana coletadas na Amazônia Brasileira. Belém: Embrapa-CPATU, 1991. 191p. (EmbrapaCPATU. Documentos, 58).

MARTEL, J.H.I.; FERRAUDO, A.S.; MÔRO, J.R.; PERECIN, D. Estatística multivariada na discriminação de raças amazônicas de pupunheiras (Bactris gasipaes Kunth.) em Manaus. Revista Brasileira de Fruticultura, v.25, p.1-9, 2003.

NASCIMENTO FILHO, F.J. do; ATROCH, A.L.; SOUSA, N.R. de; GARCIA, T.B.; CRAVO, M. da S.; COUTINHO, E.F. Divergência genética entre clones de guaranazeiro. Pesquisa Agropecuária Brasileira, v.36, p.501-506, 2001.

OLIVEIRA, M. do S.P. de. Descritores mínimos para o açaizeiro (Euterpe oleracea Mart.). Belém: Embrapa-CPATU, 1998. 3p. (Embrapa-CPATU. Pesquisa em andamento, 205).

OLIVEIRA, M. do S.P. de; FARIAS NETO, J.T. de. Cultivo do açaizeiro para produção de frutos. In: NOGUEIRA, O.L.; OLIVEIRA, M. do S.P. de; FARIAS NETO, J.T.; ROGEZ, H.L.G. Açaí: manejo, produção e processamento. Fortaleza: Instituto Frutal, 2006. p.14-67.

OLIVEIRA, M. do S.P. de; FERREIRA, D.F.; SANTOS, J.B. dos. Seleção de descritores para a caracterização de germoplasma de açaizeiro para produção de frutos. Pesquisa Agropecuária Brasileira, v.41, p.1133-1140, 2006.

OLIVEIRA, M. do S.P. de; LEMOS, M.A.; SANTOS, E.O. dos; SANTOS, V.F. dos. Variação fenotípica em acessos de açaizeiro (Euterpe oleracea Mart.) para caracteres relacionados à produção de frutos. Belém: Embrapa-CPATU, 1998. 23p. (Embrapa-CPATU. Boletim de pesquisa, 209).

RIBEIRO, F.E.; SOARES, A.R.; RAMALHO, M.A.P. Divergência genética entre populações de coqueiro-gigante-do-brasil. Pesquisa Agropecuária Brasileira, v.34, p.1615-1622, 1999.

ROHLF, F.J. Numerical taxonomy and multivariate analysis system. Version 2.1. New York: Exeter Software, 2000. 38p.

SAS INSTITUTE (Cary, Estados Unidos). SAS language and procedures: usage. Version 8.1. Cary, 2000. 1 CD-ROM.

SOUSA, N.R. Variabilidade genética e estimativas de parâmetros genéticos em germoplasma de guaranazeiro. 2003. 99p. Tese (Doutorado) - Universidade Federal de Lavras, Lavras.

SUGIMURA, Y.; ITANO, M.; SALUD, C.D.; OTSUJI, K.; YAMAGUCHI, H. Biometric analysis on diversity of coconut palm: cultivar classification by botanical and agronomical traits. Euphytica, v.98, p.29-35, 1997.

VARGAS, A.; BLANCO, F.A. Fruit characterization of Cocos nucifera L. (Arecaceae) cultivars from the Pacific coast of Costa Rica and the Philippines. Genetic Resources and Crop Evolution, v.47, p.483-487, 2000.

VICENTE, M.C. de; GUZMÁN, F.A.; ENGELS, J.; RAMANATHA RAO, V. Genetic characterization and its use in decision making for the conservation of crop germplasm. In: THE ROLE OF BIOTECHNOlOGY, 1., 2005, Turin. Proceedings. Turin, 2005. p.121-128. 